\title{
Complete information pivotal-voter model with asymmetric group size
}

\author{
Christos Mavridis $^{1} \cdot$ Marco Serena $^{2}$ (iD
}

Received: 8 March 2018 / Accepted: 2 July 2018 / Published online: 18 July 2018

(c) The Author(s) 2018

\begin{abstract}
We study the equilibria of the standard pivotal-voter participation game between two groups of voters of asymmetric sizes (majority and minority), as originally proposed by Palfrey and Rosenthal (Public Choice 41(1):7-53, 1983). We find a unique equilibrium wherein the minority votes with certainty and the majority votes with probability in $(0$, 1 ); we prove that this is the only equilibrium in which voters of only one group play a pure strategy, and we provide sufficient conditions for its existence. Equilibria where voters of both groups vote with probability in $(0,1)$ are analyzed numerically.
\end{abstract}

Keywords Costly voting $\cdot$ Pivotal voter model $\cdot$ Complete information

\section{Introduction}

Pivotal-voter models were pioneered by the seminal contribution of Palfrey and Rosenthal (1983)—henceforth, PR. They analyze a complete information setting wherein two groups of individuals, each preferring one of two alternatives, simultaneously choose between abstaining or voting for their preferred alternative. Voting is costly and the winner is decided by simple majority rule. Despite the simplicity of this pivotal-voter game, when solving for its equilibria technical difficulties and multiplicity issues arise. PR provide a partial characterization of these equilibria, some conjectures, and the analysis of two special cases. ${ }^{1}$ Nevertheless, though PR's work has been proven to be highly influential in

\footnotetext{
1 In particular, PR analyze (1) identical group sizes and symmetry of strategies across groups and (2) aggregate probabilities of voting across individuals of different groups summing to 1. Besides (1) and (2), which are special cases of our analysis, they also study two alternative and tractable settings: one assuming a status quo (ties are broken in favor of one group, instead of randomly), and another, namely "k equilibria", in which the individuals of one group mix with identical probability, whereas among the individuals of the other group, k vote with probability 1 and the remaining with probability 0 .
}

Marco Serena

marco.serena@tax.mpg.de

Christos Mavridis

c.mavridis@lancaster.ac.uk

1 Department of Economics, Lancaster University, Bailrigg, Lancaster LA1 4YX, UK

2 Max Planck Institute for Tax Law and Public Finance, Munich, Germany 
recent decades, a complete characterization of the equilibria of their original game is still missing. The work of Nöldeke and Peña (2016) — henceforth, NP_uses Bernstein polynomials to characterize the equilibria under symmetric group sizes and symmetric probabilities of voting across groups. ${ }^{2}$ In contrast, we allow the two groups of voters to be of different sizes and the voters in each group to vote with asymmetric probabilities, as initially proposed by PR.

An equilibrium is a pair of voting probabilities that the individuals in each group follow such that no individual has a profitable deviation.

NP confirm PR's conjectures about "Totally Mixed" equilibria (i.e., equilibria at which all individuals vote with the same probability $p \in(0,1))$ in the symmetric setting, namely, that individuals face a cost-of-voting threshold with the following three properties. Below the threshold, no "Totally Mixed" equilibrium exists; at the threshold, a unique "Totally Mixed" equilibrium requires that everyone votes with a probability 0.5 ; and above the threshold, there exist exactly two "Totally Mixed" equilibria, one at which everyone votes with a probability less than 0.5 and one at which everyone votes with probability greater than 0.5 .

In the present paper, the two groups are assumed to differ in size. The main result is that for sufficiently low costs of voting, there is a unique "Partially Mixed" equilibrium where the individuals in the minority (i.e., the smaller group) vote with probability 1 and the individuals in the majority (i.e., the bigger group) vote with a probability $p \in(0,1)$, which decreases in the size of the majority and increases in the size of the minority as well as in the cost of voting. This "Partially Mixed" equilibrium resembles the equilibrium of the pivotal-voter model with private information on the cost of voting-see Taylor and Yildirim (2010) — where members of the minority vote with a strictly greater probability than those of the majority do. This result is often called the "underdog effect." 3 Thus, in the "Partially Mixed" equilibrium, on the one hand, the minority has a higher individual probability of voting, but, on the other hand, it is composed of fewer members. We shed light on that tradeoff by providing sufficient conditions for the minority's preferred alternative to be more likely to win than that of the majority. The characterization of this equibrium as well as the equilibria of this model in general are of particular interest to experimental studies of voter turnout, since the theoretical predictions of the model have been tested against behavior observed in the lab. See, for instance, experimental work based on the PR model in Schram and Sonnemans (1996) and Palfrey and Pogorelskiy (forthcoming).

The remainder of the paper is structured as follows. In Sect. 2 we describe PR's original voter participation game. In Sect. 3 we analyze the equilibria, examining whether they entail pure strategies played by the individuals belonging to both groups (Sect. 3), one group only (Sect. 4), or no group (Sect. 5). Section 6 concludes.

\section{Model}

Consider a complete information setting with two groups of individuals of size $m$ and $n$, with $m, n \in \mathbb{N}_{+}$. Throughout the paper, we assume that $m>n>1$. The analysis of $n=1$ is ruled out to avoid having to deal with trivial cases. The analysis of $m=n$ produces

\footnotetext{
${ }^{2}$ Demichelis and Dhillon (2010) provide some heuristic approximations to test PR's conjectures when the population grows large and group sizes are symmetric.

${ }^{3}$ Laboratory experiments under private information confirm the underdog effect, e.g., Levine and Palfrey (2007).
} 
different results-see NP. We use subindex $i \in\{m, n\}$ to identify the group. The individuals are called upon to cast votes between two alternatives, $M$ and $N$. An $m$-individual (i.e., an individual of group $m$ ) prefers alternative $M$, and an $n$-individual (i.e., an individual of group $n$ ) prefers alternative $N$. That is, if $M(N)$ wins, the benefit of an $m-(n-)$ individual equals 1 ; otherwise, it equals 0 . If an individual casts a vote, she faces a cost of voting, $c>0$. Thus, the payoff for an individual when her preferred alternative wins is $1-c$ if she voted, and 1 if she did not vote. Individuals simultaneously choose whether to vote for their preferred alternative or abstain, since voting for the non-preferred alternative is strictly dominated. The winning alternative is decided by majority rule, and ties are broken by a fair coin toss.

Each $i$-individual chooses the probability of voting, denoted by $p_{i}$, that maximizes her expected payoff, given the choices of all other individuals. We consider Quasi-Symmetric Nash Equilibria (QSNE), that is, all $i$-individuals follow the same equilibrium strategy $p_{i}^{*}$. Besides being used in PR, ${ }^{4}$ the QSNE has been exploited in private-information pivotalvoter models to obtain that individuals adopt cut-off strategies regarding the cost of voting (e.g., Börgers 2004; Taylor and Yildirim 2010).

A pair $\left(p_{i}^{*}, p_{j}^{*}\right)$ is a QSNE if an $i$-individual does not want to deviate from $p_{i}^{*}$ if she expects every other $i$ - individual likewise to play $p_{i}^{*}$ and all $j$-individuals to play $p_{j}^{*}$. A QSNE can be of one of the following three types:

1. "Pure" (Sect. 3) $\left(p_{m}^{*}, p_{n}^{*}\right) \in\{0,1\}^{2}$,

2. "Partially Mixed" (Sect. 4) $p_{m}^{*} \in\{0,1\}, p_{n}^{*} \in(0,1)$ or $p_{m}^{*} \in(0,1), p_{n}^{*} \in\{0,1\}$, or

3. "Totally Mixed" (Sect. 5) $\left(p_{m}^{*}, p_{n}^{*}\right) \in(0,1)^{2}$.

Define $A_{i}$ with $i \in\{m, n\}$ as the probability that the vote of an $i$-individual is pivotal. Then ${ }^{5}$

$$
\begin{aligned}
A_{m}= & \sum_{s=0}^{n}\left(\begin{array}{c}
m-1 \\
s
\end{array}\right)\left(\begin{array}{c}
n \\
s
\end{array}\right) p_{m}^{s}\left(1-p_{m}\right)^{m-s-1} p_{n}^{s}\left(1-p_{n}\right)^{n-s} \\
& +\sum_{s=0}^{n-1}\left(\begin{array}{c}
m-1 \\
s
\end{array}\right)\left(\begin{array}{c}
n \\
s+1
\end{array}\right) p_{m}^{s}\left(1-p_{m}\right)^{m-s-1} p_{n}^{s+1}\left(1-p_{n}\right)^{n-s-1}
\end{aligned}
$$

and

$$
\begin{aligned}
A_{n}= & \sum_{s=0}^{n-1}\left(\begin{array}{c}
m \\
s
\end{array}\right)\left(\begin{array}{c}
n-1 \\
s
\end{array}\right) p_{m}^{s}\left(1-p_{m}\right)^{m-s} p_{n}^{s}\left(1-p_{n}\right)^{n-s-1} \\
& +\sum_{s=0}^{n-1}\left(\begin{array}{c}
m \\
s+1
\end{array}\right)\left(\begin{array}{c}
n-1 \\
s
\end{array}\right) p_{m}^{s+1}\left(1-p_{m}\right)^{m-s-1} p_{n}^{s}\left(1-p_{n}\right)^{n-s-1}
\end{aligned}
$$

\footnotetext{
${ }^{4}$ PR's definition of QSNE allows for asymmetries across group members if they adopt pure strategies. In other words, only players playing a totally mixed strategy (i.e., $\left.p_{i} \in(0,1)\right)$ are supposed to follow the same equilibrium strategy $p_{i}^{*}$; some players within the same group who play pure strategies play $p_{i}^{*}=0$ and others play $p_{i}^{*}=1$. Such mixing gives rise to what PR call "k-equilibria."

${ }^{5}$ Notice that, in order to lighten the notation, we use $A_{i}$ rather than $A_{i}\left(m, n, p_{m}, p_{n}\right)$.
} 
We now explain how expression (1) is constructed. A single $m$-individual, who computes her probability of being pivotal, takes as given the voting probabilities $\left(p_{m}, p_{n}\right)$ of all other individuals. The $m$-individual is pivotal when her vote either breaks a tie or when it creates one. In (1), the first summation is the voter's probability of breaking a tie, and the second of creating a tie. She can break a tie with her vote if the number of $m$-individuals who vote equals the number of $n$-individuals who vote. Let us call that number $s$. Of the $m-1$ other $m$-individuals, exactly $s$ vote with probability $\left(\begin{array}{c}m-1 \\ s\end{array}\right) p_{m}^{s}\left(1-p_{m}\right)^{m-s-1}$. On the other hand, out of $n n$-individuals, exactly $s$ vote with probability $\left(\begin{array}{c}n \\ s\end{array}\right) p_{n}^{s}\left(1-p_{n}\right)^{n-s}$. The second summation of (1) is constructed similarly: an $m$-individual can create a tie with her vote if the number of $m$-individuals who vote (which is again called $s$ ) is one less than the number of $n$-individuals who vote. Expression (2) is the analogous expression for an $n$-individual.

An $i$-individual casts a vote if her expected utility from casting the pivotal vote is greater than her cost of voting. Since ties are broken by a fair coin toss, if the vote of a pivotal individual creates (breaks) a tie, her expected utility increases from 0 to $1 / 2$ (from $1 / 2$ to 1 ). In both cases, the increase in utility is $1 / 2$. Thus, the condition for an $i$-individual to vote reads

$$
A_{i} \geq 2 c \text {. }
$$

\section{3 "Pure" equilibria}

If $A_{i}<2 c$ or $A_{i}>2 c$, then an $i$-individual respectively abstains or votes with certainty (i.e., pure strategy). If $A_{i}=2 c$, the $i$-individuals are indifferent between voting or not (i.e., mixed strategy). Therefore, $2 c$ can be interpreted as the minimum probability of being pivotal such that an $i$-individual will vote. For that reason, if $c>1 / 2$ no individual votes in equilibrium. This turns out to be true even if $c=1 / 2$. We formalize these results in the following proposition.

Proposition 1 If $c \geq 1 / 2$, a unique QSNE exists. It is given by $p_{m}^{*}=p_{n}^{*}=0$.

Proof Fix $i \in\{m, n\}$ and let $p_{i}^{*}>0$. First, if $c>1 / 2$, by (3) we have $A_{i}>1$, which is a contradiction, since $A_{i}$ is a probability. Second, if $c=1 / 2$, by (3) $A_{i}=1$. Then $p_{i}^{*} \notin(0,1)$ because otherwise no $i$-individual would be pivotal with certainty, so $A_{i}<1$, which leads to a contradiction. Therefore, we need to rule out only $c=1 / 2$ and $p_{i}^{*}=1$, which we do in the remainder of the proof. To do so we need to distinguish the following cases:

Case 1 If $p_{m}^{*}=1$, then alternative $M$ wins regardless of $p_{n}^{*}$ because $m>n$. Then, no $n$-individual would want to incur the cost of voting, so $p_{n}^{*}=0$. Thus, for a single $m$-individual a deviation to $p_{m}=0$ would be profitable, leading to a contradiction.

Case 2 If $p_{n}^{*}=1$, then it is necessary that $A_{n} \geq 2 c=1$, and thus $A_{n}=1$. This is the case if the $n$-individuals are certain that either $n$ or $n-1$ of the $m$-individuals vote. However, that cannot happen either if $p_{m}^{*} \in(0,1)$ (because it would imply a stochastic number of votes cast by the $m$-group) or if $p_{m}^{*}=\{0,1\}$ (because it would imply $m$ or 0 votes cast by the $m$-group). Thus, we reach a contradiction.

The previous proposition shows that if the cost of voting is high enough, the only equilibrium that exists is the "Pure" one in which nobody votes. In the remainder of the paper we analyze the more interesting case of a lower cost of voting $(c<1 / 2)$, so that individuals 
may vote with positive probability. The result causes strategic interactions that will generate multiple equilibria.

We conclude this section proving that when $c<1 / 2$ no "Pure" equilibria exist.

Proposition 2 For $c<1 / 2$, there exists no "Pure" QSNE.

Proof Fix $i \in\{m, n\}$ and $j \neq i$. Assume that $p_{i}^{*}=0$. Then $p_{j}^{*}=0$ cannot be a QSNE because $A_{j}=1$ so a deviation to voting for a $j$-individual would be profitable. Also $p_{j}^{*}=1$ cannot be a QSNE because a deviation to abstention of a $j$-individual would not affect the outcome of the election and save her cost of voting. Finally, $p_{m}^{*}=p_{n}^{*}=1$ cannot be a QSNE because the $n$-individuals lose for sure and they would thus be better-off not voting.

Having completely characterized the QSNE when $c \geq 1 / 2$ (Proposition 1) and having ruled out any "Pure" QSNE when $c<1 / 2$ (Proposition 2), we are left to analyze "Partially Mixed" and "Totally Mixed" equilibria when $c<1 / 2$.

\section{4 "Partially Mixed" equilibria}

The next Proposition establishes the existence of a unique "Partially Mixed" equilibrium at which the members of the minority (i.e., the $n$-individuals) vote with certainty. Note that PR do not analyze this case. ${ }^{6}$

Proposition 3 A $\hat{c}<1 / 2$ exists such that: if $c>\hat{c}$, no "Partially Mixed" QSNE is possible, and if $c \leq \hat{c}$, there exists a unique "Partially Mixed" QSNE. The latter is given by $p_{n}^{*}=1$ and $p_{m}^{*} \in(0,1)$ which is decreasing in $m$ and increasing in $n$ and $c$.

Proof Fix $i \in\{m, n\}$ and $j \neq i$.

Step 1 We show that there is no "Partially Mixed" QSNE that involves the members of one group abstaining, $p_{i}^{*}=0$, and members of the other group playing a mixed strategy, $p_{j}^{*} \in(0,1)$. If $i$-individuals abstain, a $j$-individual is pivotal only if none of her groupmates happen to vote. Thus, we can write the probabilities of being pivotal as $A_{j}=\left(1-p_{j}\right)^{j-1}$ and $A_{i}=\left(1-p_{j}\right)^{j}+j p_{j}\left(1-p_{j}\right)^{j-1}$.

In order to sustain any such "Partially Mixed" QSNE, it has to be that $A_{i} \leq 2 c$ and $A_{j}=2 c$ and, thus, $A_{i} \leq A_{j}$, or equivalently

$$
\left(1-p_{j}\right)^{j}+j p_{j}\left(1-p_{j}\right)^{j-1} \leq\left(1-p_{j}\right)^{j-1}
$$

Since $p_{j} \in(0,1)$, we can divide the above condition by $\left(1-p_{j}\right)^{j-1}$ and obtain $j \leq 1$, which is a contradiction.

Step 2 We show that no "Partially Mixed" QSNE exists that involves the members of the majority voting with certainty: $p_{m}^{*}=1$ and $p_{n}^{*} \in(0,1)$. Since $m>n$, the $m$-group wins

\footnotetext{
${ }^{6}$ In particular, in Sect. 5 of PR, cases 1 to 7 consider situations of status quo biased rules or cases wherein only a fraction of the members of a certain group vote with certainty. In PR's notation, the missing case, which we analyze, would be $k=N$ and $M>N$ (which PR denote as R1).
} 

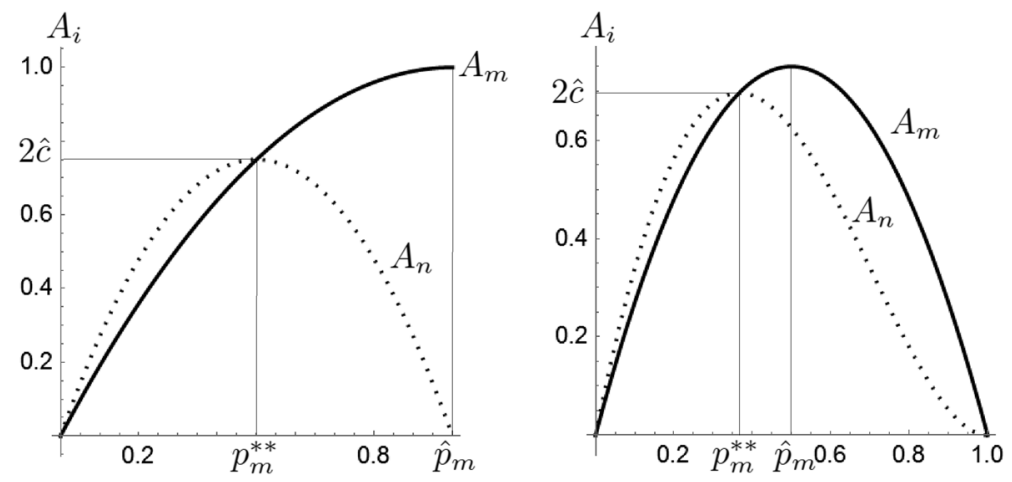

Fig. $1 A_{m}$ (solid line) and $A_{n}$ (dotted line) as a function of $p_{m}$, on the left for $(m, n)=(3,2)$ and on the right for $(m, n)=(4,2)$

with certainty, so the $n$-individuals are better off abstaining $\left(p_{n}^{*}=0\right)$ so as to save the cost $c$ without affecting their preferred alternative's probability of victory.

Step 3 The last case to analyze is that of a "Partially Mixed" QSNE with $p_{n}^{*}=1$ and $p_{m}^{*} \in(0,1)$. We then have

$$
A_{m}=\left(\begin{array}{c}
m-1 \\
n
\end{array}\right) p_{m}^{n}\left(1-p_{m}\right)^{m-n-1}+\left(\begin{array}{c}
m-1 \\
n-1
\end{array}\right) p_{m}^{n-1}\left(1-p_{m}\right)^{m-n}
$$

and

$$
A_{n}=\left(\begin{array}{c}
m \\
n-1
\end{array}\right) p_{m}^{n-1}\left(1-p_{m}\right)^{m-n+1}+\left(\begin{array}{c}
m \\
n
\end{array}\right) p_{m}^{n}\left(1-p_{m}\right)^{m-n}
$$

In order to sustain any such "Partially Mixed" QSNE, it has to be that $A_{m}=2 c$ and $A_{n} \geq 2 c$. Thus, $A_{m} \leq A_{n}$.

We divide the remainder of the proof of this proposition into two steps. We will use Fig. 1 to provide the intuition behind the two steps.

In Step 3.1, we consider $A_{m} \leq A_{n}$ and show that it holds if and only if $p_{m} \leq p_{m}^{* *} \in[0,1]$ with a unique $p_{m}^{* *}$. In Step 3.2, we consider $A_{m}=2 c$ and show that: (i) $A_{m}=0$ if $p_{m}=0$, (ii) the unique maximum of $A_{m}$ in $p_{m} \in[0,1]$ occurs at $\hat{p}_{m}$, and (iii) $\hat{p}_{m}>p_{m}^{* *}$. Those properties can be seen in Fig. 1, which helps follow the proof. Thus, in the interval wherein the "Partially Mixed" QSNE must lie, namely, $p_{m} \in\left(0, p_{m}^{* *}\right]$ (see Step 3.1), $A_{m}$ increases in $p_{m}$. Therefore, setting $\hat{c}=\left.\frac{1}{2} A_{m}\right|_{p_{m}=p_{m}^{* *}}$ provides the unique cut-off value for $c$ below (above) which a "Partially Mixed" equilibrium does (not) exist. The result thus follows.

Step 3.1 Consider $A_{m} \leq A_{n}$. Multiply both sides by $p_{m}^{1-n}\left(1-p_{m}\right)^{1+n-m}$ to obtain

$$
\left(\begin{array}{c}
m-1 \\
n
\end{array}\right) p_{m}+\left(\begin{array}{c}
m-1 \\
n-1
\end{array}\right)\left(1-p_{m}\right) \leq\left(\begin{array}{c}
m \\
n-1
\end{array}\right)\left(1-p_{m}\right)^{2}+\left(\begin{array}{c}
m \\
n
\end{array}\right) p_{m}\left(1-p_{m}\right)
$$

Divide by $(m-1)$ ! and multiply by $n !(m-n+1)$ ! both sides of the inequality and obtain 


$$
\begin{aligned}
& (m-n)(m-n+1) p_{m}+n(m-n+1)\left(1-p_{m}\right) \\
& \quad \leq n m\left(1-p_{m}\right)^{2}+m(m-n+1) p_{m}\left(1-p_{m}\right) \\
& \quad \Longleftrightarrow n(m-n+1)-2 n(m-n+1) p_{m} \leq-m(m-n+1) p_{m}^{2}+m n-2 m n p_{m}+m n p_{m}^{2} \\
& \quad \Longleftrightarrow-n(n-1)+2 n(n-1) p_{m} \leq m(2 n-m-1) p_{m}^{2}
\end{aligned}
$$

which can be rewritten as:

$$
m(m-2 n+1) p_{m}^{2}+2 n(n-1) p_{m}-n(n-1) \leq 0
$$

In order to find the roots of the quadratic Eq. (5) we use a non-standard method that simplifies the computation significantly. In particular, the solution of a general equation $A x^{2}+B x+C=0$ can be written as: ${ }^{7}$

$$
x=\frac{-2 C}{B \pm \sqrt{B^{2}-4 A C}}
$$

Formula (6) is equivalent to the standard formula for finding the roots of a quadratic equation, but it is more convenient in our case because: (1) it gives one valid root when $A=0$, which could happen in our case, and (2) the root is easier to compare with $\hat{p}_{m}$, which we will discuss in Step 3.2, since they are both roots of quadratic equations with identical coefficients $B$ and $C$, but with different $A$ s.

By (6), the roots of (5) are

$$
p_{m}=\frac{2 n(n-1)}{2 n(n-1) \pm \sqrt{4 n^{2}(n-1)^{2}+4 m(m-2 n+1) n(n-1)}}
$$

Next we simplify the discriminant:

$$
\begin{aligned}
4 n^{2}(n-1)^{2}+4 m(m-2 n+1) n(n-1) & =4 n(n-1)\left[n^{2}-n+m^{2}-2 m n+m\right] \\
& =4 n(n-1)(m-n)(m-n+1)
\end{aligned}
$$

and, thus,

$$
p_{m}=\frac{n(n-1)}{n(n-1) \pm \sqrt{n(n-1)(m-n)(m-n+1)}}
$$

\footnotetext{
7 The formula is used in Muller's method and is based on Vieta's formula. See Muller (1956). A simple way to verify the formula is to note that

$$
\left(\frac{-B \pm \sqrt{B^{2}-4 A C}}{2 A}\right)\left(\frac{B \pm \sqrt{B^{2}-4 A C}}{-2 C}\right)\left(\frac{-2 C}{B \pm \sqrt{B^{2}-4 A C}}\right)=\frac{-2 C}{B \pm \sqrt{B^{2}-4 A C}}
$$

That is, the standard formula for the roots of a quadratic equation times 1 yields (6). We thank an anonymous referee for suggesting this straightforward way of validating the formula.
} 
The root with "+" in (7) belongs to the interval $[0,1]$. If $m-2 n+1>0$, the root with "-" is negative and the function defined by (5) is convex; thus, (5) is satisfied at all $p_{m}$ s smaller than its root with "+". If $m-2 n+1<0$, the root with "-" is greater than 1 and the function defined by (5) is concave; thus, once again, (5) is satisfied at all $p_{m}$ s smaller than its root with "+". If $m=2 n-1, p_{m}^{* *}=\frac{1}{2}$ trivially and the function defined by (5) is increasing. Thus $p_{m} \leq p_{m}^{* *}$, with

$$
p_{m}^{* *}=\frac{n(n-1)}{n(n-1)+\sqrt{n(n-1)(m-n)(m-n+1)}}
$$

concluding Step 3.1 of the proof.

Step 3.2 The fact that $A_{m}=0$ if $p_{m}=0$ is trivial since $p_{n}^{*}=1$. Consider $A_{m}=2 c$. Call $\hat{p}_{m}$ the maximum of the function $A_{m}$ in $p_{m} \in[0,1]$, which we will prove to be unique. In order to compute $\hat{p}_{m}$, take the derivative of $A_{m}$ with respect to $p_{m}$, which equals 0 if and only if

$$
\begin{aligned}
& \left(\begin{array}{c}
m-1 \\
n
\end{array}\right)\left[n p_{m}^{n-1}\left(1-p_{m}\right)^{m-n-1}-(m-n-1) p_{m}^{n}\left(1-p_{m}\right)^{m-n-2}\right]+ \\
& +\left(\begin{array}{c}
m-1 \\
n-1
\end{array}\right)\left[(n-1) p_{m}^{n-2}\left(1-p_{m}\right)^{m-n}-(m-n) p_{m}^{n-1}\left(1-p_{m}\right)^{m-n-1}\right]=0 \\
& \quad \Longleftrightarrow(m-n)\left[n p_{m}\left(1-p_{m}\right)-(m-n-1) p_{m}^{2}\right]+ \\
& +n\left[(n-1)\left(1-p_{m}\right)^{2}-(m-n) p_{m}\left(1-p_{m}\right)\right]=0 \\
& \quad \Longleftrightarrow-(m-n)(m-n-1) p_{m}^{2}+n(n-1)\left(1-p_{m}\right)^{2}=0 \\
& \quad \Longleftrightarrow(2 n-m)(m-1) p_{m}^{2}-2 n(n-1) p_{m}+n(n-1)=0
\end{aligned}
$$

Again, apply the quadratic formula (6), and obtain

$$
\hat{p}_{m}=\frac{-2 n(n-1)}{-2 n(n-1) \pm \sqrt{4 n^{2}(n-1)^{2}-4 n(n-1)(2 n-m)(m-1)}}
$$

If $m=2 n$, trivially $\hat{p}_{m}=\frac{1}{2}$ (see Fig. 1 , right panel), which is a case broadly analyzed in PR. The discriminant can be simplified to:

$$
\begin{aligned}
4 n^{2}(n-1)^{2}-4 n(n-1)(2 n-m)(m-1) & =4 n(n-1)\left[n^{2}-n-2 m n+2 n+m^{2}-m\right] \\
& =4 n(n-1)(m-n)(m-n-1)
\end{aligned}
$$

and, thus,

$$
\hat{p}_{m}=\frac{n(n-1)}{n(n-1)+\sqrt{n(n-1)(m-n)(m-n-1)}}
$$


where we discarded the root with " + " in front of the square root in (8) because it is not in $[0,1] .{ }^{8}$ Notice that $m=n+1 \Longrightarrow \hat{p}_{m}=1$, as can be seen in Fig. 1 .

A straightforward comparison of $\hat{p}_{m}$ and $p_{m}^{* *}$ yields $\hat{p}_{m}>p_{m}^{* *}$, and the reasoning before Step 3.1 concludes the proof of the first part of the proposition.

We are left to show the comparative statics of $p_{m}^{*}$ with respect to $c, m, n$. We proved already that $A_{m}$ increases in $p_{m}$ in the relevant interval $p_{m} \in\left(0, p_{m}^{* *}\right]$ and, thus, the equilibrium $p_{m}^{*}$, which solves $A_{m}=2 c$, increases in $c$. By the same argument, it is sufficient to conclude the comparative statics exercise to show that $A_{m}$ increases if one substitutes $m+1$ for $m$, and that it decreases if one substitutes $n+1$ for $n$. The former condition coincides with inequality $A_{m} \leq A_{n}$, which has to hold in a "Partially Mixed" equilibrium, whereas the latter condition is equivalent to:

$$
\begin{aligned}
& \left(\begin{array}{c}
m-1 \\
n
\end{array}\right) p_{m}^{n}\left(1-p_{m}\right)^{m-n-1}+\left(\begin{array}{c}
m-1 \\
n-1
\end{array}\right) p_{m}^{n-1}\left(1-p_{m}\right)^{m-n} \\
& \quad \geq\left(\begin{array}{c}
m-1 \\
n+1
\end{array}\right) p_{m}^{n+1}\left(1-p_{m}\right)^{m-n-2}+\left(\begin{array}{c}
m-1 \\
n
\end{array}\right) p_{m}^{n}\left(1-p_{m}\right)^{m-n-1} \\
& \quad \Longleftrightarrow\left(\begin{array}{c}
m-1 \\
n-1
\end{array}\right) p_{m}^{n-1}\left(1-p_{m}\right)^{m-n} \geq\left(\begin{array}{c}
m-1 \\
n+1
\end{array}\right) p_{m}^{n+1}\left(1-p_{m}\right)^{m-n-2} \\
& \quad \Longleftrightarrow n(n+1)\left(1-p_{m}\right)^{2} \geq(m-n)(m-n-1) p_{m}^{2} \\
& \quad \Longleftrightarrow m(m-2 n-1) p_{m}^{2}+2 n(n-1) p_{m}-n(n-1) \leq 0
\end{aligned}
$$

where the last inequality is similar to (5). In fact, the only difference is that the coefficient of the term $p_{m}^{2}$ is now smaller and (5) thus implies (9), which concludes the proof.

In the "Partially Mixed" QSNE we found, the minority votes with certainty and the majority plays a mixed strategy. Thus, on the one hand, the minority is composed of fewer members than the majority, but, on the other hand, each of them votes with greater probability (in fact, with certainty) than the members of the majority. Which of these two effects is stronger is an interesting question that tells us which of the two alternatives $\{M, N\}$ is more likely to win. In the following proposition, we provide sufficient conditions for the latter effect to dominate the former.

Proposition 4 In the "Partially Mixed" QSNE the preferred alternative of the minority (n-group) is more likely to win than that of the majority (m-group) if at least one of these conditions hold:
1. $m p_{m}^{*}$ is an integer
2. $c \rightarrow 0$
3. $n=k m$ with $k \in(0,1)$ and $m \rightarrow \infty$

\footnotetext{
${ }^{8}$ In particular, the root with " + " in front of the square root in (8) is negative if $m>2 n$ and greater than 1 if $m<2 n$.

9 To see this, note that $A_{n}=A_{m}$ if the latter is evaluated at $m+1$.
} 
Proof The number of votes cast by the $n$-individuals equals $n$, and the number of votes cast by the $m$-individuals follows a binomial distribution $\operatorname{Bi}\left(m, p_{m}^{*}\right)$

1. If $m p_{m}^{*}$ is an integer, then the median of the distribution is exactly $m p_{m}^{*}$, and, thus, the claim is identical to $m p_{m}^{*}<n$. For that to hold, it suffices to show that $m p_{m}^{* *}<n$ because we know from the above analysis that $p_{m}^{*} \in\left(0, p_{m}^{* *}\right]$ in the "Partially Mixed" QSNE.

$$
\begin{aligned}
& m p_{m}^{* *}<n \\
& \Longleftrightarrow \frac{m n(n-1)}{n(n-1)+\sqrt{n(n-1)(m-n)(m-n+1)}}<n \\
& \Longleftrightarrow m(n-1)<n(n-1)+\sqrt{n(n-1)(m-n)(m-n+1)} \\
& \Longleftrightarrow(m-n)(n-1)<\sqrt{n(n-1)(m-n)(m-n+1)} \\
& \Longleftrightarrow(m-n)(n-1)<n(m-n+1) \\
& \Longleftrightarrow m n-n^{2}-m+n<m n-n^{2}+n
\end{aligned}
$$

which trivially holds true.

2. We next prove that the claim holds under the alternative assumption that $c \rightarrow 0$. The expected number of votes cast by $m$-individuals increases in $p_{m}^{*}$ since it is the outcome of a binomial distribution $\operatorname{Bi}\left(m, p_{m}^{*}\right)$, and from Proposition 3 we know that $p_{m}^{*}$ increases in $c$; thus, the expected number of votes cast by $m$-individuals increases in $c$. Also, the number of votes cast by $n$-individuals equals $n$ and, thus, it does not change in $c$. We conclude the argument by showing that for $c$ sufficiently small $(c \rightarrow 0)$ the expected number of votes cast by $m$-individuals is less than $n$, so that the claim follows.

If $c \rightarrow 0$, the mixing condition for $m$-individuals dictates that $A_{m}=2 c$; thus, $A_{m} \rightarrow 0$. Since $p_{n}^{*}=1$, the only possibility for $A_{m} \rightarrow 0$ is that $p_{m}^{*} \rightarrow 0$, which implies that the minority wins with certainty by casting exactly $n$ votes.

3. First, we show the limit of $p_{m}^{* *}$ as $m$ goes to infinity.

$$
\begin{aligned}
\lim _{m \rightarrow \infty} p_{m}^{* *} & =\lim _{m \rightarrow \infty} \frac{k m(k m-1)}{k m(k m-1)+\sqrt{k m(k m-1)(m-k m)(m-k m+1)}} \\
& =\frac{k^{2}}{k^{2}+\sqrt{k^{2}(1-k)^{2}}} \\
& =k
\end{aligned}
$$

Since in the "Partially Mixed" QSNE $p_{m}^{*} \leq p_{m}^{* *}$ we multiply both sides by $\frac{m}{n}$, and then take the limit as $m$ goes to infinity:

$$
\lim _{m \rightarrow \infty} \frac{m p_{m}^{*}}{n} \leq \lim _{m \rightarrow \infty} \frac{m p_{m}^{* *}}{n}=\frac{k}{k}=1 .
$$

Therefore, we have that

$$
\lim _{m \rightarrow \infty} \frac{m p_{m}^{*}}{n} \leq 1 .
$$


Define $X_{m}$ to be the random variable determining the number of votes cast by group $m$. That variable follows the binomial distribution $\operatorname{Bi}\left(m, p_{m}^{*}\right)$ and as $m$ goes to infinity the distribution of random variable $\frac{X_{m}-n p_{m}^{*}}{\sqrt{n p_{m}^{*}\left(1-p_{m}^{*}\right)}}$ converges to a standard normal distribution $N(0,1)$, meaning that as $m$ goes to infinity the distribution of $X_{m}$ converges to the normal distribution $N\left(n p_{m}^{*}, n p_{m}^{*}\left(1-p_{m}^{*}\right)\right)$. In a normal distribution the mean and median coincide. As such (10) implies that as $m$ goes to infinity group $n$ is more likely to win than group $m$.

The three sufficient conditions of Proposition 4 are strong, but while condition 1 is purely technical, conditions 2 and 3 mirror some interesting real-life applications. Condition 2 resembles situations in which the cost of voting is negligible. One example is when the time needed to cast a vote is insignificant, as it is often the case for online or postal voting. Another example is when the ballot box is very close to every individual, as in case of small firms or academic departments. Condition 3 approximates the case of large electorates such as national elections or referendums. The use of a fixed proportion between $m$ and $n$ (i.e., $k$ ), provides us with a tractable setting to analyze such large electorates.

While the three conditions are sufficient they are not necessary for the result. A counterexample is the following. Let $m=70$ and $n=45$. In the "Partially Mixed" equilibrium we have $A_{m}=2 c$ and $A_{n} \geq 2 c$, which when $c \cong 0.0979$ can sustain an equilibrium with $p_{m}^{*}=\frac{198-3 \sqrt{1430}}{133}$ and $p_{n}^{*}=1$. This equilibrium gives rise to group $m$ winning with probability approximately equal to 0.5445 .

\section{5 "Totally Mixed" equilibria}

Propositions 1, 2, and 3 completely characterized the "Pure" and "Partially Mixed" equilibria of the pivotal voter model. In this section we examine numerically the "Totally Mixed" equilibria, which may exist only when $c<1 / 2$. In any such equilibrium the voting conditions (3) for the two groups hold with equality. That is, the mixing condition for the $m$-individuals is

$$
A_{m}=2 c
$$

and that of the $n$-individuals is

$$
A_{n}=2 c
$$

In a QSNE all individuals within a group adopt the same strategy. Thus, in order to analyze the "Totally Mixed" equilibria we look for pairs $\left(p_{m}, p_{n}\right) \in(0,1)^{2}$ that satisfy both mixing conditions (11) and (12). In particular, we focus on the $p_{m}$ solving (11) for $p_{n} \in(0,1)$ and on the $p_{n}$ solving (12) for $p_{m} \in(0,1)$. The intersections between those two sets in the space $\left(p_{m}, p_{n}\right) \in(0,1)^{2}$ are the "Totally Mixed" equilibrium pairs $\left(p_{m}^{*}, p_{n}^{*}\right)$, which are what we study in this section.

The "Totally Mixed" case_-in contrast to the "Pure" and "Partially Mixed" casesentails solving a system of two polynomial equations of an arbitrarily large degree-expressions (11) and (12) — and, thus, it presumably is impossible to find an algebraic solution for equilibrium strategies. As such, while we found a number of partial characterizations of the space on which the solutions to those polynomials lie, we failed to complete the analysis, 

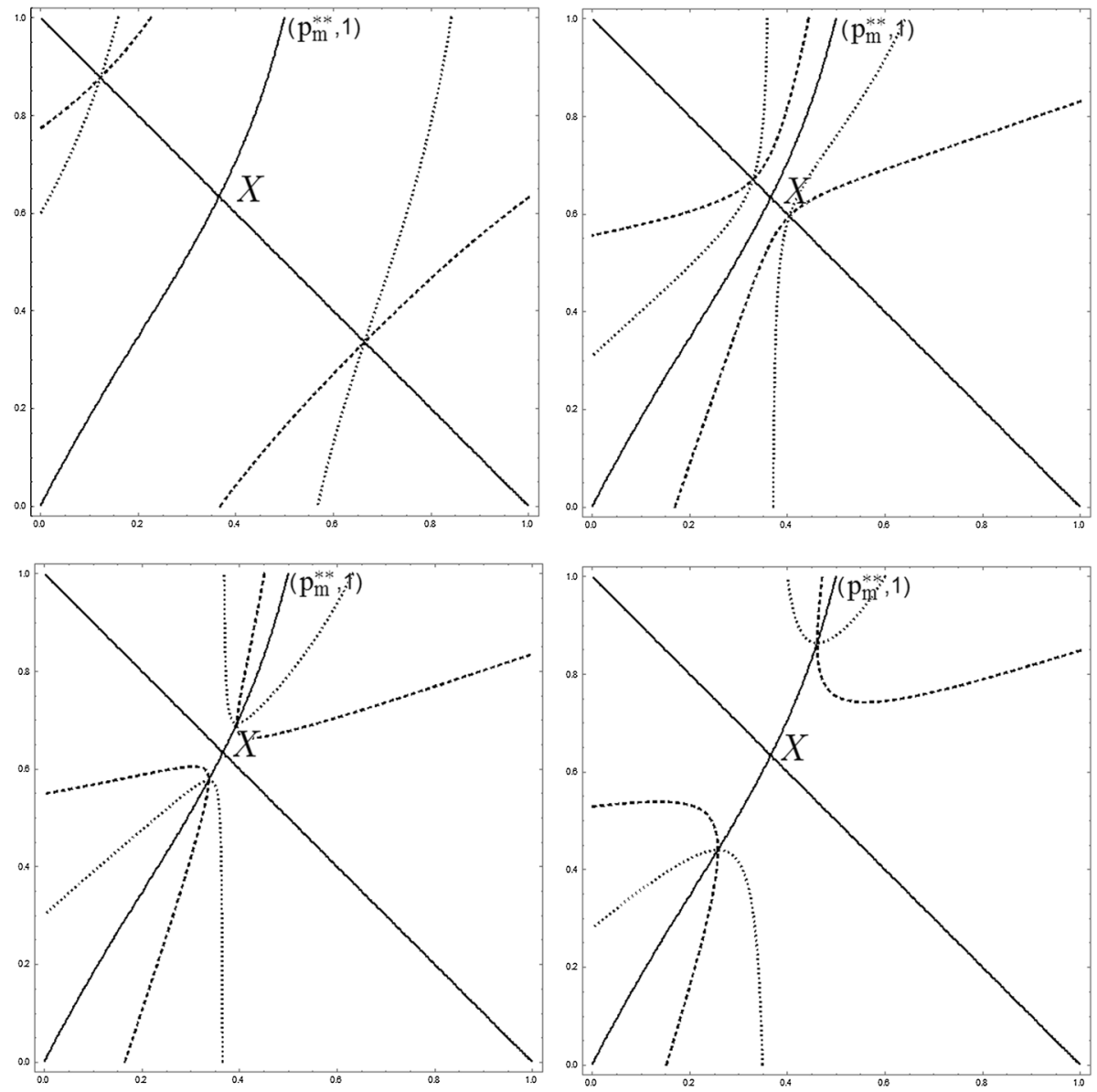

Fig. 2 Set of points in the $\left(p_{m}, p_{n}\right)$-space where $A_{m}=A_{n}$ (solid line), $A_{m}=2 c$ (dashed line) and $A_{n}=2 c$ (dotted line) when $m=3$ and $n=2$, and from left to right, from top to bottom, $c=0.2,0.345,0.349,0.36$

which therefore remains an open research question. For that reason, we describe in what follows some numerical evidence on "Totally Mixed" equilibria.

Conditions (11) and (12) require $A_{m}=A_{n}$. The set of pairs $\left(p_{m}, p_{n}\right) \in(0,1)^{2}$ at which $A_{m}=A_{n}$ is depicted in black in Fig. 2 for the special case of $m=3$ and $n=2$. That set turns out to be composed of a downward-slopping line, namely $p_{m}+p_{n}=1$, which does not depend on $(m, n)$, and an upward-slopping line, which starts at $(0,0)$, corresponds to $p_{n}=p_{m}$ when $m=n,{ }^{10}$ and becomes steeper as $m$ increases. In fact, the upward-sloping line touches the upper bound of the unit box at point $\left(p_{m}^{* *}, 1\right) .{ }^{11}$ Any "Totally Mixed" equilibrium must be on one of those two lines.

\footnotetext{
${ }^{10}$ In the case of $m=n$ analyzed in NP, the upward-sloping line corresponds to $p_{m}=p_{n}$.

11 The closed form solution of $p_{m}^{* *}$ is found in the proof of Proposition 3. In fact, $p_{m}^{* *}$ is the solution to $A_{m}=A_{n}$ when $p_{n}=1$.
} 
If $c<1 / 2$, it turns out that at most two "Totally Mixed" equilibria exist. ${ }^{12}$ We now provide a description of how these equilibria behave as $c$ changes. Consider Fig. 2, where the crossing between the upward-sloping and downward-sloping lines is labeled $X$, we change $c$ and plot the two mixing conditions (dotted and dashed lines). For small values of $c$, the two equilibria lie on the downward-sloping line close to points $(0,1)$ and $(1,0)$. As $c$ increases, the two equilibria move towards point $X$ along the downward-sloping line, they reach $X$, and then they move along the upward-sloping line (one upwards and one downwards) towards $(0,0)$ and $\left(p_{m}^{* *}, 1\right)$, which they reach for sufficiently large $c$. Thus, the equilibria, when moving along the downward sloping line, have the property that the equilibrium voting probabilities move in the opposite direction, which is diametrically opposed to what NP show.

\section{Conclusions}

Palfrey and Rosenthal (1983) proposed a seminal pivotal-voter model involving two groups of individuals, each preferring one of two alternatives. They provided only a partial characterization of the equilibria, leaving the complete characterization for future research. While Nöldeke and Peña (2016) analyze the equilibria of Palfrey and Rosenthal (1983) restricting their attention to the case of symmetric group sizes, we contribute to the equilibrium analysis of the pivotal-voter model under asymmetric group sizes, as originally proposed by Palfrey and Rosenthal (1983). We deem the asymmetric case particularly interesting because it us allows to embrace several applications. In fact, the vast majority of the real-life voting situations involve asymmetric group sizes: left- and right-wing supporters in elections, or members of a firm's board of directors voting on an issue, or, in general, members of committees favoring of or opposing the status quo. These, and many other examples, arise in which it is natural to discuss election outcomes when the number of supporters of one alternative differs from that of the other alternative.

Acknowledgements Open access funding provided by Max Planck Society. We are grateful to Luis Corchón and Ignacio Ortuño-Ortín for their advice and support. We would like to thank Kai Konrad, Diego Moreno, Antoine Loeper, Georg Nöldeke, Nikolas Tsakas, Jan Zapal, two referees and an associate editor for their useful comments as well as participants in seminars in Universidad Carlos III de Madrid, the Max Planck Institute for Tax Law and Public Finance, PET 2015 in Luxemburg, the MOMA Meeting 2016, the 2016 EPCS meeting, the 2nd Lancaster Game Theory Conference and the 16th Conference on Research on Economic Theory and Econometrics. Marco Serena thanks the network MOMA under project ECO201457673-REDT. All errors are our own.

Open Access This article is distributed under the terms of the Creative Commons Attribution 4.0 International License (http://creativecommons.org/licenses/by/4.0/), which permits unrestricted use, distribution, and reproduction in any medium, provided you give appropriate credit to the original author(s) and the source, provide a link to the Creative Commons license, and indicate if changes were made.

\footnotetext{
12 The analysis that PR carry out for two special cases makes them "[...] conjecture that the class of all totally quasi-symmetric equilibria is much larger than those we have been able to investigate." However, our numerical simulations show that the class of equilibria admits, at most, two equilibria even in the original asymmetric setting.
} 


\section{References}

Börgers, T. (2004). Costly voting. American Economic Review, 94(1), 57-66.

Demichelis, S., \& Dhillon, A. (2010). Learning in elections and voter turnout. Journal of Public Economic Theory, 12, 871-896.

Levine, D., \& Palfrey, T. (2007). The paradox of voter participation? A laboratory study. American Political Science Review, 101(1), 143-158.

Muller, D. E. (1956). A method for solving algebraic equations using an automatic computer. Mathematical Tables and other Aids to Computation, 10, 208-215.

Nöldeke, G., \& Peña, J. (2016). The symmetric equilibria of symmetric voter participation games with complete information. Games and Economic Behavior, 99, 71-81.

Palfrey, T. R., \& Pogorelskiy, K. (forthcoming). Communication among voters benefits the majority party. Economic Journal. https://doi.org/10.1111/ecoj.12563.

Palfrey, T. R., \& Rosenthal, H. (1983). A strategic calculus of voting. Public Choice, 41(1), 7-53.

Schram, A., \& Sonnemans, J. (1996). Voter turnout as a participation game: An experimental investigation. International Journal of Game Theory, 25, 385-406.

Taylor, C. R., \& Yildirim, H. (2010). A unified analysis of rational voting with private values and groupspecific costs. Games and Economic Behavior, 70(2), 457-471. 